$W-E 1$

\title{
Double resonance and four wave mixing FEL studies of InAs/GaAs quantum dots and local modes in ionic crystals
}

\author{
C. R. Pidgeon ${ }^{\mathrm{a}}$, J-P. Wells ${ }^{\mathrm{a}, \mathrm{b}}$, I. V. Bradley ${ }^{\mathrm{a}, \mathrm{b}}$ and B. N. Murdin ${ }^{\mathrm{c}}$ \\ ${ }^{\text {a} P h y s i c s ~ D e p a r t m e n t, ~ H e r i o t ~ W a t t ~ U n i v e r s i t y, ~ E d i n b u r g h ~ E H 14 ~ 4 A S, ~ U K ~}$ \\ [tel: 0131451 3022; fax: 0131451 3136; email: c.r.pidgeon@hw.ac.uk] \\ 'FOM Institute 'Rijnhuizen', P.O. Box 1207, NL-3430 BE Nieuwegein, The Netherlands \\ 'Physics Department, University of Surrey, Guildford GU2 5XH, UK
}

\section{Introduction}

A brief review is given of lifetime and line broadening studies with the free electron laser at FOMRijnhuizen (FELIX), emphasising work on far infrared (FIR) modulated photoluminescence (PL) of bound-to-bound transitions in InAs/GaAs quantum dots [1] and mid-infrared (MIR) four wave mixing experiments on localised modes of $\mathrm{H}^{-}$ions in calcium fluoride crystals [2]. We have made new far/near infrared double resonance measurements of self-assembled InAs/GaAs quantum dots. The FIR resonance is unambiguously associated with a bound-bound intraband transition in the neutral dots, and analysis of the FIR double resonance linewidth enables us to show that the inhomogeneous broadening of the PL cannot be attributed solely to size and composition fluctuation. In addition we have made time resolved studies of local modes in ionic crystals, where non-radiative decay plays an important role in the optical pumping cycle of laser gain media. We have made pump-probe studies of the local modes created upon the introduction of a light impurity, in particular the $\mathrm{H}$ - ion, in $\mathrm{CaF}_{2}$ in the spectral region 700 to $1200 \mathrm{~cm}^{-1}$. We have also measured the free induction decay of this mode using a non-colinear degenerate four wave mixing (DFWM) geometry. The observed coherent transient is striking in having large quantum beat modulations at negative time which are associated with vibrational ladder climbing.

\section{Double resonance spectroscopy of InAs/GaAs self-assembled quantum dots}

There have been many measurements of intraband spectroscopy in semiconductor quantum dots as a means to probe the excited states, but we describe here a new inter/intra-band double resonance investigation of quantum dots. The interest of this technique is that unlike FIR absorption or even photo-induced FIR absorption it has allowed us to make an unequivocal assignment of the resonant electronic bound-to-bound intraband absorption simultaneously with the interband excitonic transition in neutral dots (i.e. giving the electron and hole splittings within the same dots, without the need for $n$-type and p-type samples). These transitions may be strongly dependent on charge, and it is important to study the transitions in neutral systems since these are or primary technological interest. We have applied the technique to self-organised dots grown at a low growth-rate, which show well resolved photoluminescence (PL) lines. The double resonance and its polarisation selection rule allows us to identify not only the levels involved in the PL transition but the relative importance of the causes of its inhomogeneous broadening.

In summary, we have measured the intersublevel absorption in self-assembled InAs/GaAs quantum dots, and shown that higher energy PL lines arise from the in-plane confinement [1]. We make a 
quantitative determination of the separation between both lowest electronic states and corresponding hole states $(\mathrm{E} 2-\mathrm{E} 1=55 \mathrm{meV}, \mathrm{H} 2-\mathrm{H} 1=13 \mathrm{meV})$ in a single experiment. Lastly, the measurements of the inhomogeneity of our very narrow line width sample shows that mechanisms other than spatial size or potential depth fluctuations must also contribute to the broadening of the PL spectra. Very recently we have shown that when the samples are annealed, the PL is sharper, and we now observe a spatial hole in the PL which blue shifts as the FIR is shifted to shorter wavelengths. We therefore attribute the lack of spatial holes in the unannealed samples to indium fluctuations within the dots which are removed by the anneal. We determine from the width of the spatial hole the contribution to the inhomogeneous width due to size fluctuations. We also observe a reduction in the wings of the PL characteristic of a line narrowing which we attribute to FIR induced phase-locking of the of the driven oscillator system. This has previously been inferred from transport and intraband spectroscopy but has not been measured directly in interband measurements.

\section{Dynamics of hydrogenic localised vibrational modes}

The development of near infrared solid state lasers, based around for example $3 \mathrm{~d}^{2}$ configuration ions such as $\mathrm{Cr}^{4+}$ and $\mathrm{V}^{3+}$, is hampered by heavy quenching of the optical excitation by nonradiative relaxation at room temperature. Recent studies have shown the importance of local mode vibrations of the optically active center in mediating the non-radiative multiphonon relaxation process. We report on pump-probe and free induction decay experiments on the localised vibrational modes of ionic hydrogen in $\mathrm{CaF}_{2}$ and $\mathrm{LiYF}_{4}$ crystals using the FELIX output in the ten micron region. These systems are attractive for their simplicity, the only possible non-radiative path being into the lattice band modes [2]. Only one time-resolved study of these systems using pulses shorter than the local mode lifetime has been reported previously, and indeed no coherent investigations have been made.

For resonant excitation of the $\mathrm{CaF}_{2}: \mathrm{H}^{-}$mode at $9.36 \mu \mathrm{m}$, a $10 \mathrm{~K}$ lifetime of $\mathrm{T}_{1}=43 \mathrm{ps}$ has been determined by a three beam pump-probe technique. The temperature dependence of the decay rate fits well to a three phonon anharmonic decay process. In addition, we have observed giant quantum coherence effects in the localised modes. MIR free induction decay experiments show large modulations at negative delay times due to interference between multiple vibrational levels. Spectrally resolving the degenerate four wave mixing signal allows unambiguous assignment of the participating vibrational states. The dependence of the signal intensity upon the delay path between the exciting free electron laser pulses, and in particular the quantum beats at negative times, can be accounted for theoretically in terms of the resonant third order polarisation with a common dephasing time for the excited states. The fit was obtained by adjusting the dephasing rate to fit the positive delay signal, giving $\mathrm{T}_{2}=8.0 \pm 0.5 \mathrm{ps}$. This value corresponds closely to the linewidth of the spectrally resolved infrared free induction decay, and is consistent with the $T_{1}$ value (43ps) obtained from the pump-probe measurement.

[1] B N Murdin, A R Hollingworth, D Clark, P C Findlay, C R Pidgeon, J-P Wells, I V Bradley, S Malik and R Murray, Phys. Rev. B to be published (2000).

[2] J-P Wells, C W Rella, I V Bradley, I Galbraith and C R Pidgeon, Phys. Rev. Lett. $\underline{84}, 4998$ (2000). 